\title{
G-protéines oncogéniques et cancers des glandes endocrines
}

\section{Les nouvelles \\ de ce numéro ont été préparées par Pascale Briand Jean-Claude Dreyfus Jean-Pierre Grünfeld Axel Kahn Claude Matuchansky Marc Peschanski Hubert Vaudry*}

- Laboratoire d'endocrinologie moléculaire, Cnrs URA 650, unité affiliée à l'Inserm, université de Rouen, 76134 Mont-Saint-Aignan, France.
Nous avons déjà rapporté, il y a environ un an, les résultats de l'équipe de H.R. Bourne, de San Francisco (CA,USA), concernant les mutations de la sous-unité $\alpha_{\mathrm{s}}$ de la G-protéine stimulatrice dans des tumeurs hypophysaires sécrétant de l'hormone de croissance chez l'homme (syndrome d'acromégalie) [1]. Ces mutations altéraient l'activité GTP-asique de la G-protéine stimulant l'adénylate cyclase, conduisant à une activation permanente de ce système. Or, au niveau des cellules somatotropes de l'hypophyse, le récepteur de la somatolibérine (GHRH, growth hormone releasing hormone), une hormone de croissance pour ces cellules, est couplé à l'adénylate cyclase via cette protéine $G_{s}$. De tels systèmes où un récepteur hormonal intervenant dans la stimulation de la croissance d'une cellule cible est couplé à un système effecteur via une G-protéine sont la règle dans les tissus glandulaires endocrines. H.R. Bourne et ses collègues ont donc cherché à déterminer si d'autres tumeurs endocrines pouvaient être expliquées par des mutations de la sous-unité régulatrice $\alpha$ des G-protéines hétérotrimériques de forme $\alpha \beta$ $\gamma$. L'hypothèse à la base de l'étude entreprise était que les deux mutations récemment détectées au niveau de $\alpha_{\mathrm{s}}$ (Gly ${ }^{227} \rightarrow \operatorname{Arg}$ et $\operatorname{Arg}^{201} \rightarrow$ Cys ou His) identifiaient des acides aminés particulièrement importants pour le contrôle de l'activité GTP-asique de toutes les sous-unités $\alpha\left(\alpha_{\mathrm{i}}, \alpha_{\mathrm{o}} \ldots\right.$ et autres). De fait, ces résidus sont extraordinairement conservés au cours de l'évolution des espèces et l'Arg ${ }^{201}$ est la cible de l'ADP ribosylation catalysée par la toxine cholérique, modification qui entraîne une stimulation permanente des protéines $G_{S}$ de la muqueuse intestinale et explique ainsi l'hypersécrétion d'eau et de sel à la base du syndrome cholérique.

Des amorces oligonucléotidiques permettant d'amplifier par PCR la séquence nucléotidique des gènes des différentes sous-unités $\alpha$ connues, séquence incluant les acides aminés conservés, ont donc été synthétisées et utilisées pour tester l'intégrité de cette région protéique dans un grand nombre de tumeurs des glandes endocrines.

Une mutation de l'Arg ${ }^{179}$ de la sous-unité $\alpha_{\mathrm{i} 2}$ (les protéines $\mathrm{G}_{\mathrm{i}}$, ou inhibitrices, sont connues pour inhiber l'adénylate cyclase lorsqu'elles sont ellesmêmes activées) a été observée dans 3 tumeurs corticosurrénaliennes sur 11 testées et dans 3 tumeurs endocrines ovariennes sur 10. De plus, sur 42 tumeurs hypophysaires avec hypersécrétion d'hormone de croissance à nouveau explorées, 18 (43\%) possédaient une mutation de la sous-unité $\alpha_{s}, 16$ fois à la position $\mathrm{Arg}^{201}$ et 2 fois à la position Gly ${ }^{227}$. Dans tous les cas, les cellules non tumorales (les globules blancs) ne possédaient que les allèles non mutés, prouvant bien l'origine somatique des mutations, limitées au tissu tumoral [2].

Contrairement aux résultats positifs rapportés ci-dessus, aucune altération 
des G-protéines $\alpha_{\mathrm{i}}$ ou $\alpha_{\mathrm{s}}$ ne fut notée dans des cancers non endocriniens (sein, colon, mélanomes, par exemple). Les résultats concernant la mutation de la sous-unité $\alpha_{\mathrm{i}}$, et non $\alpha_{\mathrm{s}}$, dans des tumeurs ovariennes et corticosurrénaliennes, sont étonnants car on considérait plutôt, jusqu'à aujourd'hui, que la LH (luteinizing hormone) et l'ACTH (adrenocorticotropin hormone) stimulaient la croissance de leurs cibles cellulaires par l'intermédiaire d'une activation de l'adénylate cyclase.

Les observations rapportées ici suggèrent que, physiologiquement, c'est plutôt l'inhibition de la synthèse de l'AMP cyclique qui peut être associée à la stimulation de la prolifération des cellules de la granulosa ovarienne et de la corticosurrénale.

Quoi qu'il en soit, les gènes codant pour les sous-unités $\alpha_{\mathrm{s}}$ et $\alpha_{\mathrm{i} 2}$ peuvent en effet être considérés comme des oncogènes en puissance, appelés respectivement gsp et gip2.

A. $\mathbf{K}$.

1. Landis CA, Master SB, Spada A, Pace AM, Bourne HR, Vallar L. GTPase inhibition mutations activate the $\alpha$ chain of $\mathrm{G}_{\mathrm{s}}$ and stimulate adenyl cyclase in human pituitary tumors. Nature $1989 ; 340: 692-6$. 2. Lyons J, Landis CA, Harsh G, et al. Two $\mathrm{G}$-protein oncogenes in human endocrine tumors. Science $1990 ; 249: 655-9$.

\section{JOURNAL DE PHARMACIE CLINIQUE}

\section{4 numéros par an vient de paraître}

Vol. 9. $n^{\circ} 3$ - septembre 1990 d. John Libbey

6, rue Blanche, 92120 MONTROUGE

\section{BRÈVES $\square$}

Maladie de CreutzfeldtJakob et mutations des prions (suite). Un des problèmes pathologiques les plus passionnants sur lequel $\mathrm{m} / \mathrm{s}$ revient souvent est celui des maladies transmissibles " à virus lents ", qui possèdent en même temps une composante génétique. Celle-ci est prouvée dans la maladie de Gerstmann-Sträussler $\left(m / s n^{\circ} 1\right.$, vol. $6, p .77)$ dans laquelle le gène qui code pour les prions (localisé sur le chromosome 20) subit des mutations, dont la plus fréquente aboutit à un changement pro-leu au codon 102 de la protéine. Dans la maladie de Creutzf eldt-jakob, dont on connaît des formes familiales, les résultats sont beaucoup moins clairs. Une équipe de Bethesda (MD, USA) [1] a trouvé dans plusieurs familles originaires d'Europe de l'Est (Grèce, Pologne, Slovaquie) et dans deux cas sporadiques une mutation du codon 200 (glutamine $\rightarrow$ lysine) a l'état hétérozygote, que l'on peut reconnaître car elle supprime une coupure par l'enzyme de restriction Bsm A1. Cette mutation était absente dans d'autres familles atteintes, chez 28 des 30 cas sporadiques testés, chez 29 autres malades neurologiques et 102 témoins. Il est donc permis de faire l'hypothèse qu'une telle mutation entraîne de façon dominante, les symptômes de la maladie, ou tout au moins en favorise l'apparition. Il convient toutefois d'être très prudent dans les conclusions, comme en tout ce qui touche les encéphalopathies spongiformes, et nous voudrons à ce sujet rappeler deux exemples. Le premier concerne une autre mutation dans le même gène, décrite par la même équipe [2] et $m / s n^{\circ} 6$, vol. 5 , p. 429), un passage méthionine $\rightarrow$ valine au codon 129 de la protéineprion, qui, à l'état homozygote cette fois, était associée à des cas de Kuru et de Creutzfeldt-Jakob. Or, dans le travail actuel [1], aucune allusion n'est plus faite à la mutation 129 , alors qu'un autre groupe [3] vient de montrer que cette mutation est fréquente dans certaines populations et représente un polymorphisme non pathogène. Le deuxième exemple est fourni par un éditorial [4] du 7 juillet dans Lancet, accompagné d'un article [5], qui tendent à élargir considérablement le cadre des encéphalopathies génétiques sur la base de l'analyse du gène, même si manquent les symptômes anatomopathologiques considéré jusqu'à présent comme spécifiques. Ces articles ont soulevé une levée de boucliers dans un numéro suivant [6] et la question demeure controversée.

[1. Goldfarb LG, et al. Lancet 1990 ; 336 : 174-5.]

[2. Goldfarb LG, et al. Am J Hum Genet 1989 ; 45 (suppl) : A189.]

[3. Owen F, et al. Am J Hum Genet 1990 ; 46 : 1215-6.]

[4. Éditorial, Lancet $1990 ; 336: 11$. [5. Collinge J, et al. Lancet 1990 ; $336: 7-9$.

[6. Letters to the Editor. Lancet $1990 ; 336: 369-70$.

\section{FLASH}

La myopathie facio-scapulo-humérale, dont l'hérédité est autosomique dominante, était la dernière des dystrophies musculaires progressives importantes dont la localisation chromosomique restait inconnue. Un groupe néerlandais vient de montrer par des méthodes de liaison sur 10 familles hollandaises que le gène responsable est situé sur le chromosome 4.

[Wijmenga $C$, et al. Lancet $1990 ; 336: 651-3$. ]
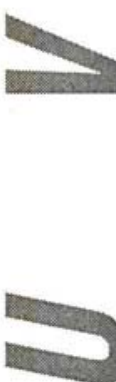

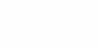

$\mathrm{m} / \mathrm{s} n^{\circ} 8$ vol. 6 , octobre 90 


\section{FLASH}

\section{UN FIL CONDUCTEUR POUR FAIRE DIFFÉRENCIER LES CELLULES MALIGNES}

Un traitement qui provoque une réversion phénotypique de la cellule maligne, un récepteur remanié par une translocation chromosomique, une voie nouvelle de découverte de médicaments contre le cancer, tels sont les fruits d'une recherche clinique coordonnée par Laurent Degos (Hôpital Saint-Louis, Paris).

L'acide tout trans-rétinoïque, dérivé de la vitamine $A$ fait différencier in vitro les cellules malignes de leucémie aiguë promyélocytaire en cellule mature. Ce dérivé est dix fois plus actif que les dérivés actuellement disponibles en thérapeutique (13 cis rétinoïde ou Roaccutane). Son effet est spécifique de la leucémie aiguë promyélocytaire. Ces résultats obtenus par Christine Chomienne et al. à l'hôpital Saint-Louis [1] trouvent tout leur intérêt dans l'efficacité thérapeutique de ce médicament. En effet, pour la première fois, un agent est capable de faire différencier des cellules malignes, in vivo. Sylvie Castaigne et al. [2] rapportent les résultats obtenus avec ce produit qu a été fabriqué d'abord en Chine, puis par Roche France. Des rémissions complètes sont obtenues avec un changement progressif de l'aspect des cellules malignes. Dans le cas de traitement de premières rechutes de cette maladie, plus de $90 \%$ des malades atteignent une rémission complète, le plus souvent à domicile, avec un simple traitement oral, sans perfusion, sans transfusion, sans antibiotique [3]. La coagulation intravasculaire disséminée, complication majeure de cette maladie, disparaît très vite.

Cependant un événement néfaste peut survenir, l'augmentation des globules blancs qui, d'une part prouve l'absence d'effet cytotoxique de ce médicament et qui, d'autre part, oblige parfois à adjoindre une chimiothérapie réductrice.

La translocation chromosomique spécifique de cette maladie $t(15 ; 17)$ a été l'objet d'une étude particulière. L'équipe de Christine Chomienne avec Hugues de Thé ldu groupe de Anne Dejean) a remarqué que le gène du récepteur $\alpha$ de l'acide rétinoïque est remanié dans cette maladie et donc doit être impliqué dans la translocation [4]. Deux équipes ont cloné cette translocation [5, 6]. Une de ces équipes (Hugues de Thé et Anne Dejean à I'Institut Pasteur) a montré que le gène du récepteur $\alpha$ de l'acide rétinoïque sur le chromosome 17 est tronqué avant le $2^{e}$ exon et se rattache à un gène myl (myéloïd leukemia) sur le chromosome 15. Une équipe anglaise vient de rapporter des résultats de même ordre [5]. Un nouveau transcript formé de la fusion de ces deux gènes est présent dans cette maladie [6].

Chez la plupart des patients étudiés, I'un et/ou l'autre des deux gènes est altéré, généralisant cette observation. Le récepteur de l'acide rétinoïque altéré par la translocation conserve ses régions d'attache à l'ADN et à l'acide rétinoïque, mais aurait acquis des propriétés nouvelles. Ainsi le ligand, l'acide rétinoïque, est capable de reprogrammer la cellule afin qu'elle se différencie et apparaisse normale, alors que le récepteur lui-même est remanié et que le gène du récepteur a acquis de nouvelles régions promotrices : un dilemme à élucider. Quoiqu'il en soit, voici le premier modèle de traitement efficace in vivo par différenciation de la cellule maligne et un fil conducteur au niveau moléculaire pour comprendre le mécanisme d'action du produit différenciateur. Ce fil d'Ariane peut ouvrir un champ nouveau de recherche pour des médicaments agissant contre le cancer en reprogrammant la cellule maligne. Hlstoire à suivre.

Laurent Degos

1. Chomienne C, Ballerini P, Balitrand N, Daniel MT, Fenaux $P$, Castaigne s, Degos L. All-trans retinoic in actue promyelocytic leukemias. II. In vitro studies: structure-function relationship. Blood (novembre 1990).

2. Castaigne S, Chomienne C, Daniel MT, Ballerini P, Berger R, Fenaux P, Degos L. All-trans retinoic acid as a differentiation therapy for acute promyelocytic leukemia. I. Clinical Results. Blood (novembre 1990).

3. Degos L, Chomienne C, Daniel MT, Berger R, Dombret H, Fenaux P, Castaigne S. Treatment of first relapse of acute promyelocytic leukaemia using all trans retinoic acid: a model for differentiation therapy. Lancet (in press).

4. Chomienne $C$, Ballerini $P$, Huang ME, Castaigne $S$, Fenaux $P$, Tiollais $P$, Dejean A, Degos $L$, de Thé $H$. The retinoic acid receptor alpha gene is rearranged in retinoic acid sensitive promyelocytic leukemia (soumis).

5 . Borrow J, Goddard AD, Sheer D, Solomon E. Molecular analysis of acute promyelocytic leukemia breakpoint cluster region on chromosome 17. Science 1990; $249: 1577-80$.

6. De The H, Chomienne C, Lanotte M, Degos L, Dejean A. The $t(15 ; 17)$ translocation of acute promyelocytic leukemia fuses the retinoic acid receptor $\alpha$ gene to a novel transcribed locus. Nature loctobre 1990). 